\title{
Smoking in pregnancy: final thoughts
}

\author{
Robert L Goldenberg, Lorraine V Klerman, Richard A Windsor, \\ H Pennington Whiteside, Jr
}

This volume highlights both good news and bad news about smoking during pregnancy. The good news is that many women quit spontaneously either just before or soon after becoming pregnant; pregnancy specific counselling with appropriate print materials results in additional cessation; and the percentage of women who smoke throughout pregnancy continues to decline. The bad news is that, each year, additional adverse impacts of maternal smoking on the fetus and infant are reported; one in five to one in six pregnant women smoke throughout their pregnancies; many pregnant smokers do not report their smoking status to their obstetric providers; most women who stop smoking during pregnancy relapse soon after childbirth; and there is no obvious powerful anti-smoking intervention appropriate for pregnant women on the horizon. Also, most of the tobacco use in pregnancy is concentrated among the poorest women-those with other adverse health behaviours, those who are the most heavily addicted, and those who often have the least psychosocial resources to overcome this addictive behaviour.

In an attempt to develop effective smoking cessation methods useful in pregnancy, the Robert Wood Johnson Foundation and its Smoke-Free Families program funded the 11 programs described in this volume, attempting to evaluate methods to increase cessation among pregnant smokers. We learned a number of lessons from observing these projects. First, many women smokers did not admit they were smoking on entry into care, thereby decreasing the number of women available for each of the studies. Second, the seismic shift in the medical care system during the project period, with many women changing from hospital and health department provider systems to private care, resulted in fewer smoking women being available for cessation studies than in the past. The managed care organisations that now served many of these women were less willing than the clinics to test experimental smoking cessation programs. Third, many of the hospital and health department clinics that had for many years provided a relatively stable environment for patients were in disarray because of decreased funding owing to loss of patients and other types of financial cutbacks. They were further burdened by additional required services such as human immunodeficiency virus (HIV) testing and counselling. Together, these left staff little time for smoking cessation efforts.

\section{Interventions}

The grantees used a wide range of often novel techniques that they hypothesised would increase smoking cessation and decrease relapse. The interventions took place in homes and in clinical sites. They used one-on-one counselling in person and by telephone. The messages were delivered by health care providers, case managers, peers, and others. Personally tailored anti-smoking messages used videos, computers, interactive voice technology, and printed guides. Messages were reinforced by financial incentives and by feedback of biochemical measures of maternal smoking and household smoke level. Despite the wide range of approaches and the innovative quality of many of them, few showed obvious benefit. However, it was usually not clear if the intervention itself did not work, or if for various reasons the intervention was not tested appropriately in the setting chosen. For example, in a number of sites, because of the work overload, many of the women apparently received only a portion of the intervention. We are left, therefore, with the question: were these types of interventions not efficacious or were they not given a fair test in the settings studied?

These interventions were considered pilot studies, thus each grantee received only $\$ 200000$ to $\$ 250000$ for a two year period. In retrospect, neither the time nor the resources were sufficient for an adequate test of the "innovative" interventions. Our process evaluation results and anecdotal reports indicated that additional time was needed for site selection, materials preparation, and pilot testing of all patient assessment and education procedures. Even then, with the chaos in the medical care systems, it is not clear if many of the studies could be implemented as planned. For example, a number of sites intended to use existing clinic staff to conduct their intervention. Initially, this approach seemed reasonable, because the goal of many projects was to incorporate the intervention into routine prenatal care so it would be self sustaining. For the reasons mentioned, this approach was often unrealistic. In most settings, health workers had a large workload of competing activities. Even if smoking cessation activities seemed appropriate, interesting, and important to the staff, they were often such low priority that they did not receive the attention necessary to make them successful.

Many of the women smokers lived lives as chaotic as the clinics they attended, and had many competing problems. In the clinic, they were bombarded with health improvement 
messages on HIV and sexually transmitted disease prevention, nutrition, drug and alcohol abuse, domestic violence, breast feeding, and child care. It is not surprising that many of the women, often single and poor, did not focus on the anti-smoking messages.

While certainly not conclusive, it is our impression that data from the pilot studies suggested that feedback based on some biochemical measure of smoking, perhaps with a monetary incentive, or monetary incentives alone, may increase smoking cessation among pregnant women. Perhaps these two approaches were less likely to "get lost" in most prenatal care systems, and with appropriate counselling may increase the motivation to stop smoking in a group of women not responding to counselling alone.

\section{Future needs}

Looking to the future, major advances in smoking cessation are needed in several areas. The first is in preventing relapse among those women who stop smoking either just before or during their pregnancy. With relapse rates approaching $70 \%$, there is tremendous opportunity to reduce the overall number of smokers by helping those who have quit to stay quit. Finding the right mix of behavioural and pharmacologic interventions to assist these women in maintaining cessation in the postpartum period is crucial. The other major area where additional progress is crucial is with the heaviest, most addicted smokers. This is the subgroup among which adverse smoking related outcomes are most likely to occur, and it is precisely these women who achieve little or no cessation in pregnancy, either spontaneously or with counselling. Behavioural interventions have shown little promise in this population. Perhaps one or more of the available pharmacologic interventions will be appropriate for this group. However, to date, there is no evidence for efficacy or safety of these methods during pregnancy, and certainly, routine use should not be recommended. ${ }^{1}$ Much additional work needs to be done in this area, including a focus on prepregnancy and postpartum interventions. ${ }^{1}$

We observed that many obstetric providers did not adopt a smoking cessation intervention, often because of a lack of consensus on efficacy and a profusion of different types of interventions, each with their own proponents. One of the major breakthroughs in the last few years (aided by several meta-analyses) is a growing consensus that a 5-15 minute counselling session by a trained provider with the provision of appropriate print materials will achieve a modest but important increase in cessation in pregnant women. ${ }^{23} \mathrm{In}$ the year 2000, the surgeon general, the Agency for Healthcare Research and Quality, and the obstetrics practice committee of the American College of Obstetricians and Gynecologists will issue similar recommendations for treating pregnant smokers. This should eliminate "lack of consensus" as a reason for not implementing appropriate smoking cessation treatment during pregnancy. The adoption by these various groups of a "treatment standard" should encourage obstetric providers to "do the right thing" for their patients.

Cigarette smoking during pregnancy continues to be one of the few preventable causes of adverse pregnancy outcomes. To reduce the unnecessary maternal, fetal, and infant damage associated with smoking, we need to implement fully the cessation methodologies that do work and continue the search for other even more effective preventive and treatment interventions. The Robert Wood Johnson Foundation's Smoke-Free Families program will continue to work toward achieving these goals.

1 Benowitz NL, Dempsey DA, Goldenberg RL, et al. Workshop summary: the use of pharmacotherapies for smoking cessation during pregnancy. Tobacco Control 2000;9(suppl III):91-4.

2 Mullen PD. Smoking during pregnancy and intervention to promote cessation: a meta-analysis. In: Spangler JG, ed. Primary care: clinics in office practice. Philadelphia: WB Sanders, 1999;26:577-89.

3 Melvin CL, Dolan-Mullen P, Windsor RA, Whiteside HP, Goldenberg RL. Recommended cessation counselling for pregnant women who smoke: a review of the evidence. Tobacco Control 2000;9(suppl III): iii80-4. 